\title{
Tumour ADC measurements in rectal cancer: effect of ROI methods on ADC values and interobserver variability
}

\author{
Doenja M. J. Lambregts • Geerard L. Beets • Monique Maas • Luís Curvo-Semedo • \\ Alfons G. H. Kessels • Thomas Thywissen • Regina G. H. Beets-Tan
}

Received: 5 April 2011 /Revised: 30 May 2011 / Accepted: 22 June 2011 /Published online: 7 August 2011

(C) The Author(s) 2011. This article is published with open access at Springerlink.com

\begin{abstract}
Objectives To assess the influence of region of interest (ROI) size and positioning on tumour ADC measurements and interobserver variability in patients with locally advanced rectal cancer (LARC).

Methods Forty-six LARC patients were retrospectively included. Patients underwent MRI including DWI (b0,500,1000) before and 6-8 weeks after chemoradiation (CRT). Two readers measured mean tumour ADCs (pre- and post-CRT) according to three ROI protocols: whole-volume, single-slice or small solid samples. The three protocols were compared for differences in $\mathrm{ADC}, \mathrm{SD}$ and interobserver variability (measured as the intraclass correlation coefficient; ICC).

Results ICC for the whole-volume ROIs was excellent (0.91) pre-CRT versus good (0.66) post-CRT. ICCs were 0.53 and
\end{abstract}

D. M. J. Lambregts $\cdot$ M. Maas • T. Thywissen •

R. G. H. Beets-Tan $(\bowtie)$

Department of Radiology, Maastricht University Medical Center,

P.O. Box 5800, 6202 AZ Maastricht, The Netherlands

e-mail: r.beets.tan@mumc.nl

R. G. H. Beets-Tan

e-mail: r.beets.tan@mumc.nl

D. M. J. Lambregts · G. L. Beets · M. Maas

Department of Surgery, Maastricht University Medical Center,

P.O. Box 5800, 6202 AZ Maastricht, The Netherlands

L. Curvo-Semedo

Department of Radiology, Radiology University Clinic, Coimbra

University Hospital,

Praceta Mota Pinto/Av. Bissaya Barreto,

3000-075 Coimbra, Portugal

\section{A. G. H. Kessels}

Department of Epidemiology,

Maastricht University Medical Center,

P.O. Box 5800, 6202 AZ Maastricht, The Netherlands
0.42 for the single-slice ROIs versus 0.60 and 0.65 for the sample ROIs. Pre-CRT ADCs for the sample ROIs were significantly lower than for the whole-volume or single-slice ROIs. Post-CRT there were no significant differences between the whole-volume ROIs and the single-slice or sample ROIs, respectively. The SDs for the whole-volume and single-slice ROIs were significantly larger than for the sample ROIs.

Conclusions ROI size and positioning have a considerable influence on tumour ADC values and interobserver variability. Interobserver variability is worse after CRT. ADCs obtained from the whole tumour volume provide the most reproducible results.

Key Points

- ROI size and positioning influence tumour ADC measurements in rectal cancer

- ROI size and positioning influence interobserver variability of tumour ADC measurements

- ADC measurements of the whole tumour volume provide the most reproducible results

- Tumour ADC measurements are more reproducible before, rather than after, chemoradiation treatment

- Variations caused by ROI size and positioning should be taken into account when using $A D C$ as a biomarker for tumour response

Keywords Diffusion magnetic resonance imaging $\cdot$ Rectal neoplasms · Observer variation · Methodology Apparent diffusion coefficient

\section{Introduction}

At present, the standard treatment for patients with locally advanced rectal cancer consists of a long course of 
neoadjuvant chemoradiation treatment (CRT) followed by surgical resection. As surgery is routinely performed in each patient-regardless of the response to treatment-response evaluation after CRT has so far not been a major issue. Nowadays there is, however, a trend towards minimally invasive treatments instead of standard surgery for wellresponding patients [1-3]. Accurate response assessment then becomes relevant, as it may directly influence treatment planning. ${ }^{18}$ F-Fluorodeoxyglucose-Positron Emission Tomography (FDG-PET) and MRI have been most extensively studied for response evaluation, but these techniques suffer from limitations in the interpretation of fibrotic scar tissue and inflammation [4, 5]. Diffusion-weighted MR Imaging (DWI) is a functional imaging technique that analyses differences in the extracellular movement of water protons to discriminate between tissues of varying cellularity [6]. Different publications on DWI have shown its potentially beneficial role for the detection and characterisation of malignant tumours [7-9]. In addition, changes in tumour diffusion during and after treatment are indicative of tissue changes on a cellular level and may be used to evaluate treatment response [10, 11]. Previous studies in a variety of tumour types have suggested that quantitative interpretation of the apparent diffusion coefficient (ADC) can be used as a biomarker for response to treatment [12-15]. For rectal cancer patients specifically, a benefit for treatment response evaluation by measuring tumour ADC values before [16-19], during [16-18, 20, 21], and after chemoradiation treatment has been suggested [22, 23]. Nevertheless - as also previously pointed out in a review by Patterson et al. [10] - there is no consensus yet on the true clinical value of $\mathrm{ADC}$ measurements for response assessment in rectal cancer. This is because the available literature consists of mainly small-scale studies with conflicting results. Moreover, in most studies, DWI evaluation was only performed by a single reader and ADC measurements by a variety of methods for region of interest (ROI) placement. Whereas some authors included the whole tumour volume [17-19, 22, $24]$, others included only a single tumour slice $[16,21]$ or small tumour samples [23], which may contribute to the large variety in reported $\mathrm{ADC}$ results. It remains unclear whether ROIs for ADC measurements should ideally incorporate the entire tumour volume or only a representative tumour section. Furthermore, none of the studies focusing on rectal tumour $\mathrm{ADC}$ have addressed the issue of interobserver variability, which is a non-negligible factor when considering the use of $\mathrm{ADC}$ as a potential marker for response in clinical practice.

The purpose of the current study is to assess the influence of ROI size and positioning on interobserver variability and $\mathrm{ADC}$ values when measuring tumour $\mathrm{ADC}$ before and after chemoradiation treatment in patients with locally advanced rectal cancer. We aim to determine which method offers the most reproducible results in order to provide a reference for further studies.

\section{Materials and methods}

\section{Patients}

This study retrospectively evaluated 46 patients who were treated for locally advanced rectal cancer between 2006 and 2010. Clinical patient data were retrieved from a patient database originating from a previous imaging study approved by the local institutional review board, for which the patients provided written informed consent. Thirty-four patients were male and 12 were female. Median age was 70 years (range 49-88). Inclusion criteria consisted of [a] histologically (biopsy) proven rectal adenocarcinoma, [b] locally advanced disease, defined on primary staging T2weighted MRI by an experienced gastrointestinal radiologist as tumour in the distal rectum $(\leq 5 \mathrm{~mm}$ from the anorectal junction), threatened or involved circumferential resection margins ( $\leq 2 \mathrm{~mm}$ margin between the tumour and mesorectal fascia) and/or positive nodal stage ( $\geq 1$ suspicious nodes, i.e. $>5 \mathrm{~mm}$ in size and/or heterogeneous signal intensity and/or irregular border), [c] treatment consisting of a long course of preoperative CRT (50.4 Gy radiation+ $2 \times 825 \mathrm{mg} / \mathrm{m}^{2} /$ day capecitabine) followed by surgical resection and [d] availability of pre- and post-CRT MR imaging including DWI. Patients with non-resectable and/ or metastatic disease were excluded. Mucinous tumours are known to have a very low cellular density and will therefore exhibit high ADC values [25]. As this may bias the study results, patients with predominantly mucinous appearing tumours (identified as predominantly high signal lesions on T2-weighted MRI) were also excluded.

\section{MR imaging}

Patients did not receive bowel preparation or spasmolytics. Imaging was performed at $1.5 \mathrm{~T}$ (Intera; Philips Medical Systems, Best, The Netherlands) using a phased array body coil. All patients underwent a pre-treatment MRI for primary tumour staging and a second, restaging MRI for response evaluation 6-8 weeks after completion of CRT. The imaging protocol consisted of standard 2D T2weighted (T2W) fast spin-echo sequences (FSE) in three orthogonal directions and an axial DWI single-shot echo planar imaging sequence, according to the method of diffusion-weighted imaging with background body signal suppression (DWIBS), acquired with b-values of 0,500 and $1000 \mathrm{~s} / \mathrm{mm}^{2}$ [26]. The sequence parameters are displayed in Table 1. The axial T2W and DWI sequences were angled in identical planes and were planned perpendicular to the tumour axis as defined on sagittal MRI. ADC maps in greyscale were automatically generated at the operating system, using a monoexponential decay model including all three b-values. 
Table 1 Sequence parameters

* DWI was acquired with bvalues of 0,500 and $1000 \mathrm{~s} / \mathrm{mm}^{2}$

\begin{tabular}{lll}
\hline & T2W FSE & DWI* \\
\hline Repetition time (ms) & $3427-8456$ & 4829 \\
Echo time (ms) & $130-150$ & 70 \\
Echotrain length & 25 & 1 \\
In plane resolution (mm $\times$ mm) & $0.78 \times 1.14$ & $2.50 \times 3.11$ \\
Section thickness (mm) & $3-5$ & 5 \\
Section gap (mm) & 2 & -1 \\
No. of sections & $22-30$ & 50 \\
No. of signals acquired & 6 & 4 \\
Sensitivity encoding (SENSE) factor & - & 2 \\
Echo planar imaging (EPI) factor & - & 53 \\
Acquisition time (min) & $5.08-6.03$ & 10.37 \\
\hline
\end{tabular}

Image evaluation

The MR images were independently analysed by two radiological researchers (DMJL and TT), who performed tumour ADC measurements on the pre- and postchemoradiation images. The readers were blinded to each other's results, the clinical patient data and pathology reports. Mean tumour ADC was evaluated by manually drawing regions of interest (ROI) on the high b-value (b1000) diffusion images and copying them to the corresponding ADC map (Fig. 1). The mean ADC+ standard deviation (SD) and the number of pixels per ROI was recorded for each individual measurement. On the pre-treatment b1000 diffusion images, tumour was defined as a focal mass showing high signal intensity compared with the signal of the normal adjacent rectal wall and corresponding with the tumour (mass showing intermediate signal intensity) on the anatomical T2-weighted MRI. On the post-chemoradiation DWI, tumour was defined as focal areas of residual high signal on the b1000 images within the location of the primary tumour bed and/or corresponding with residual tumour on T2-weighted MRI (Fig. 2). The pre-treatment images were at the readers' disposal when analysing the post-treatment images, in order to compare and identify the location of the tumour. When no remaining high signal could be visualised on DWI, three sample measurements were obtained of the rectal wall at the former location of the primary tumour, of which an example is illustrated in Fig. 3.

\section{ROI protocols}

Mean tumour ADCs were measured according to three distinct ROI protocols: [a] 'Whole-volume', [b] 'Singleslice' and [c] 'Solid tumour samples'. For the whole volume method, freehand ROIs were drawn along the border of the high signal of the tumour on the b1000 images to cover the entire tumour area of each consecutive tumour-containing slice. Mean ADC (+SD) was obtained for each slice and ADC values were averaged to calculate the mean ADC of the whole tumour volume. For the single-
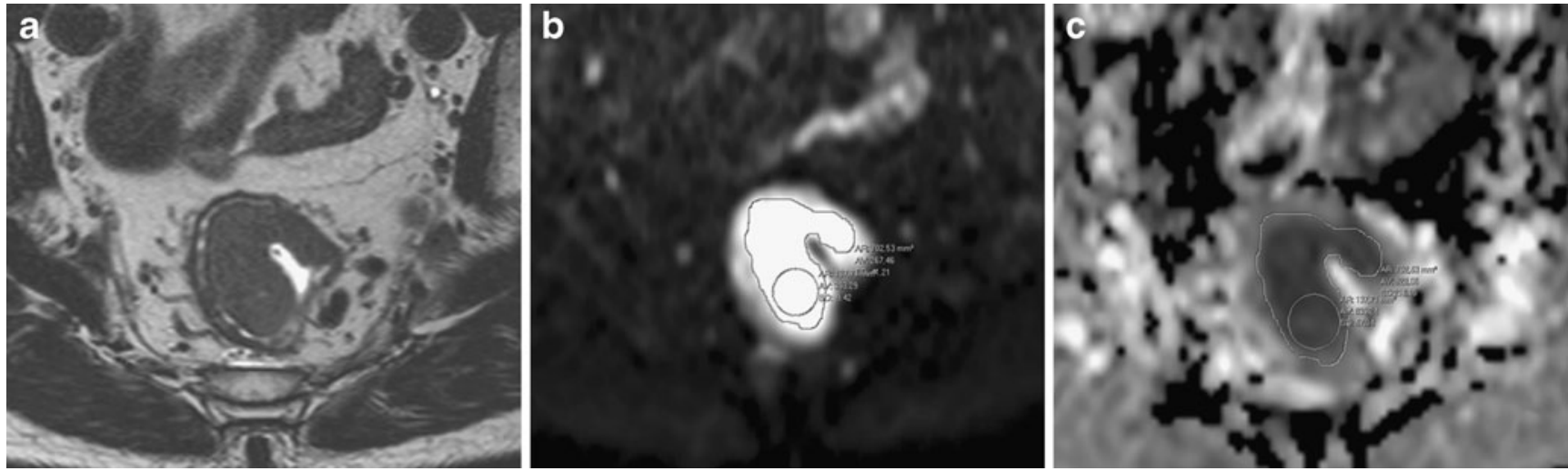

Fig. 1 Axial T2-weighted image (a), b1000 diffusion image (b) and ADC map (c) of a male patient with a tumour in the rectum. For the whole-volume and single-slice methods, ADC was measured by drawing freehand ROIs along the high signal intensity border of the tumour on the b1000 images (b) to cover the entire tumour area. ROIs were copied to the ADC map (c) to calculate ADC. For the solid sample method, tumour ADC was measured by drawing three oval- or round-shaped ROIs within the most solid tumour areas 

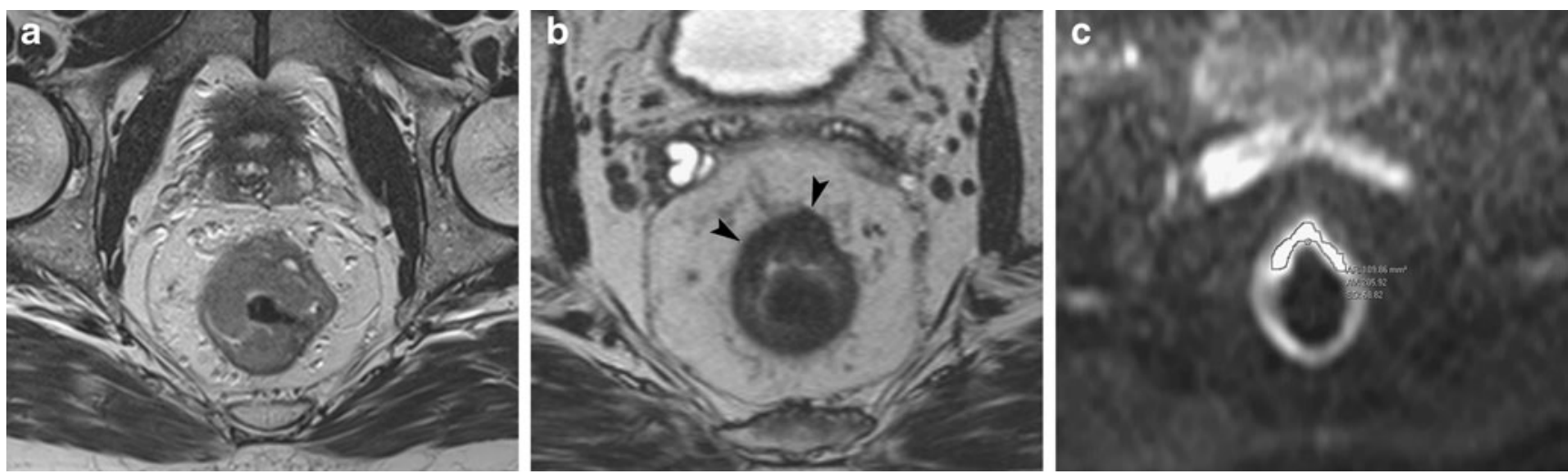

Fig. 2 Axial pre- (a) and post-treatment (b) T2-weighted images of a male patient with a rectal tumour. After treatment, the tumour has undergone mainly fibrotic changes (arrowheads). On the corresponding

slice method, a single freehand ROI was drawn in the same way (along the border of the tumour), but only on a single slice containing the largest available tumour area. For the third method, mean ADC was calculated from a sample of three round/oval-shaped ROIs that were placed within the most solid tumour part (as identified on T2W-MRI) of three independent tumour-containing slices, which an example is illustrated in Fig. 1.

Statistical analyses

Statistical analyses were performed using the Statistical Package for the Social Sciences (SPSS, version 16.0, Inc., Chicago, IL, USA). Interobserver variability for the tumour ADC measurements of the two readers for the pre- and post-CRT ADC measurements and for each individual ROI method was analysed according to the method of Bland and Altman and by calculating the intraclass correlation coefficient $(0.00-0.20$ poor, $0.21-0.40$ fair, $0.41-0.60$ moderate, $0.61-0.80$ good and $0.81-1.00$ excellent correlation). ADCs were averaged between the two observers for b1000 diffusion image, an ROI was drawn along a well-defined area of high signal intensity within the fibrosis, suggestive of residual tumour. At histology, a residual ypT2 tumour was found

further analyses. A paired samples $t$-test was used to compare [a] the pre- and post-treatment ADCs and [b] the tumour ADC values obtained by the three different ROI methods. For each patient, the average variance was calculated over the different slice measurements, weighted with the number of pixels. The mean SD for each patient was calculated as the square root of the variance. The variance (mean for the whole patient group) of the different ROI measurement methods and for the pre- and post-CRT measurements was compared using the F-statistics with the total number of slices as the degree of freedom. $P$ values $<0.05$ were considered statistically significant.

\section{Results}

Patient and treatment characteristics

Twenty-seven patients underwent a low anterior resection, 15 an abdominoperineal resection and 4 more extended surgery. At histology 6 patients had a ypT0, 5 ypT1, 14
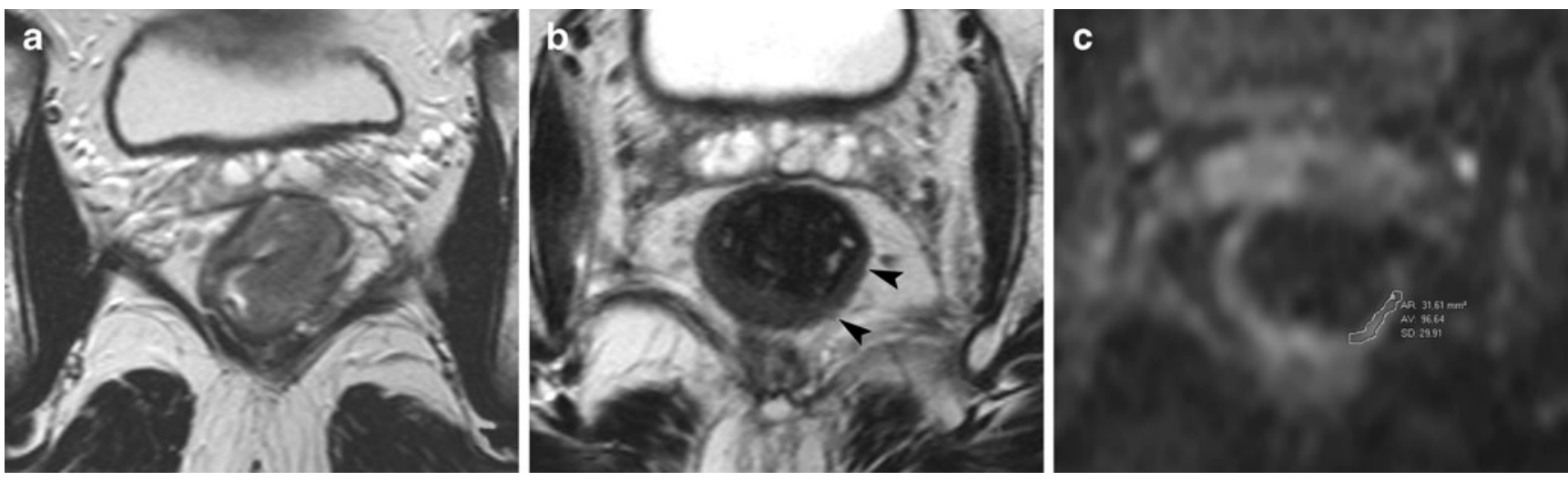

Fig. 3 Axial T2-weighted images of a male patient with a rectal tumour before (a) and after (b) chemoradiation treatment. After CRT, the rectal wall has normalised (arrowheads). On the corresponding b1000 diffusion image (c), no high signal was observed and ROIs were placed within the rectal wall at the location of the primary tumour to measure post-treatment ADC. At histology, the patient had undergone a complete response 
Table 2 Influence of choice of regions of interest (ROIs)

\begin{tabular}{lllc}
\hline & Pre-CRT & Post-CRT & $P$ \\
\hline Whole-volume ROIs & & & \\
Mean ADC $\left(* 10^{-3} \mathrm{~mm}^{2} / \mathrm{s}\right)$ & 1.10 & 1.44 & $<0.001$ \\
SD & 0.26 & 0.25 & 0.41 \\
Total ROI size $\left(\mathrm{mm}^{2}\right)$ & 7275 & 767 & $<0.001$ \\
Single-slice ROIs & & & $<0.001$ \\
Mean ADC $\left(* 10^{-3} \mathrm{~mm}^{2} / \mathrm{s}\right)$ & 1.10 & 1.48 & 0.35 \\
SD & 0.24 & 0.23 & $<0.001$ \\
Total ROI size $\left(\mathrm{mm}^{2}\right)$ & $490^{*}$ & $157^{*}$ & $<0.001$ \\
Solid sample ROIs & & & 0.14 \\
Mean ADC $\left(* 10^{-3} \mathrm{~mm}^{2} / \mathrm{s}\right)$ & $1.02^{* * * *}$ & $1.41^{* *}$ & $<0.001$ \\
SD & $0.19^{* * * *}$ & $0.20^{*}$ & $220^{* * * *}$ \\
Total ROI size $\left(\mathrm{mm}^{2}\right)$ & $696^{* * * *}$ & & \\
\hline
\end{tabular}

ypT2, 20 ypT3 and 1 a ypT4 status. Thirty-three patients had a ypN0, 9 ypN1 and 4 ypN2 status.

\section{Effect of ROI methods}

The mean tumour ADCs, SDs and total ROI sizes are displayed in Table 2 for the pre- and post-treatment measurements of each respective ROI protocol. Mean pretreatment tumour ADC was significantly lower when measured by means of small sample ROIs, compared with the whole-volume $(p<0.001)$ or single-slice protocol $(p<$ $0.001)$, respectively. For the post-CRT measurements there were no significant differences in tumour ADC between the whole-volume ROIs compared with the single-slice ( $p=$ 0.07 ) or small sample ROIs $(p=0.08)$, respectively, but the single-slice ROIs resulted in significantly higher ADCs compared with the small sample ROIs $(p=0.002)$. For the pre-CRT measurements, the variance (SD) of the small sample ROI measurements was significantly smaller than for the whole-volume ROIs $(p<0.001)$ and single-slice ROIs $(p=0.03)$, respectively. For the post-CRT measurements, the variance of the small sample ROIs was also smaller than that of the whole-volume ROIs $(p=0.003)$ and single-slice ROIs, although the latter difference was not statistically significant $(p=0.06)$. There were no significant differences in tumour ADC or variance between the wholevolume and single-slice approaches.

\section{Interobserver variability}

Intraclass correlation coefficients between the two readers are provided in Table 3 for the three ROI protocols. The interobserver reproducibility was excellent (ICC 0.91) for the pre-CRT whole-volume ADC measurements, and good (ICC 0.66) for the post-CRT measurements. For the singleslice and solid sample ROIs, the ICCs ranged from 0.42 to

0.65. Figure 4 displays the Bland-Altman plots for the whole-volume measurements performed pre- and post-CRT.

\section{Discussion}

The results of this study show that, when measuring ADC in patients with locally advanced rectal cancer, tumour ADC values and interobserver variability are highly dependent on methods of ROI analysis. ADC measurements obtained from the whole tumour volume are more reproducible than those obtained from single-slice or small sample measurements. In specific pre-treatment wholevolume ADC measurements result in excellent interobserver reproducibility.

The number and size of the ROIs affected the interobserver agreement. When comparing the different ROI protocols, the single-slice and sample ROIs resulted in considerably poorer interobserver agreement (ICC 0.42 0.65) than the whole-volume ROIs (ICC 0.66-0.91), indicating that analysing a larger number of pixels results in more reproducible ADC values. Interobserver agreement for the whole-volume ADC measurements before treatment was excellent (ICC 0.91), but results after treatment were poorer (ICC 0.66). After chemoradiation, rectal tumours

Table 3 Interobserver variability (measured as the intraclass correlation coefficient*) for the different ROI protocols

\begin{tabular}{lcc}
\hline & Pre-CRT & Post-CRT \\
\hline Whole-volume ROIs & 0.91 & 0.66 \\
Single-slice ROIs & 0.53 & 0.42 \\
Solid sample ROIs & 0.60 & 0.65 \\
\hline * 0.00-0.20 poor; 0.21-0.40 fair; $0.41-0.60$ moderate; & $0.61-0.80$ \\
good; 0.81-1.00 excellent correlation
\end{tabular}




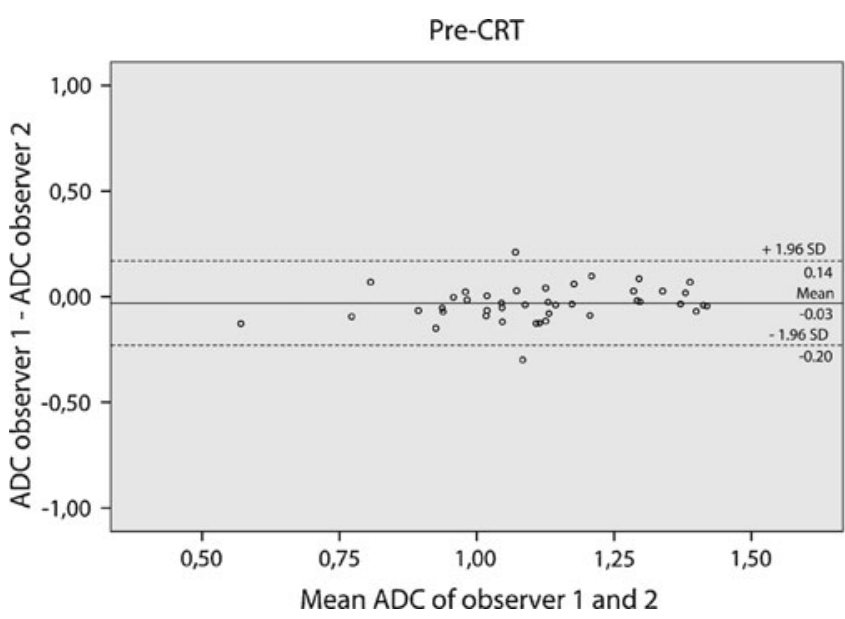

Fig. 4 Interobserver reproducibility for the whole-volume tumour ADC measurements performed pre- and post-chemoradiation treatment. BlandAltman plots of the mean ADC of the two observers (x-axis) against the difference in ADC between the two observers (y-axis). The continuous

have often undergone massive fibrotic changes and defining a region of tumour residue within the fibrosis may be more difficult (Fig. 5). In cases where the tumour has completely regressed and the bowel wall has normalised or become fibrotically thickened, it can be even more challenging to correctly define an ROI (Fig. 3). After CRT, ADC measurements thus seem to be more affected by the interpretation skills of the reader than before CRT, when the tumour is generally better defined.

The choice of ROIs also significantly influenced the tumour ADC values. On pre-CRT MRI, the whole-volume and single-slice ROIs resulted in significantly higher tumour ADC values than the small sample ROIs. The small sample ROIs only included the most viable solid tumour parts, which may explain the lower ADC values. In

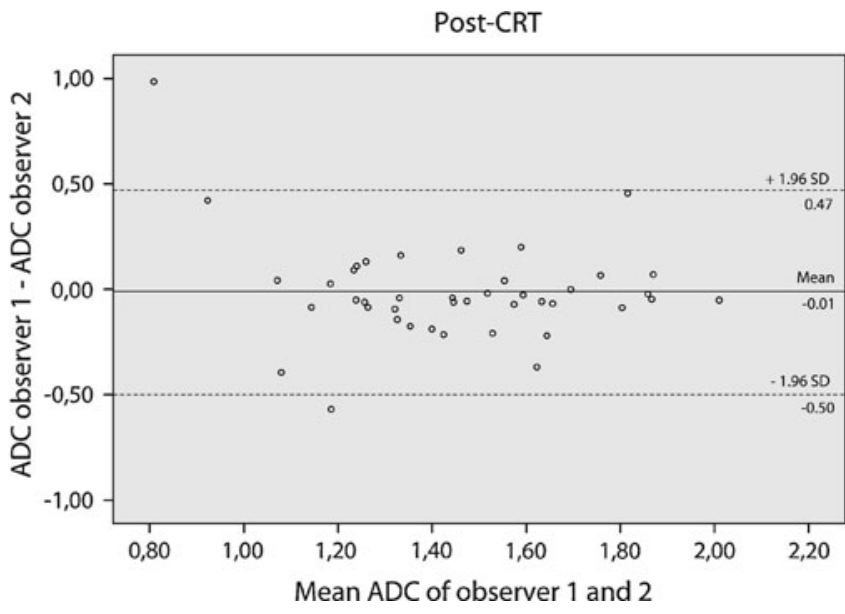

lines represent the mean absolute difference (bias) in ADC between the two observers; the dashed lines represent the $95 \%$ confidence intervals of the mean differences (limits of agreement)

this setting, areas of necrosis are likely to be excluded from the ADC measurements, while the presence of necrosis before onset of treatment is in fact believed to be an important indicator when aiming at evaluating response. A previous study of Roth and co-authors showed that wholevolume tumour ADC measurements were a better predictor of response than ROIs chosen only from viable regions of the tumour [18]. Although the focus in their study was on perfusion $\mathrm{CT}$ in patients with colorectal cancer Goh et al. also found that, when obtaining pharmacokinetic parameters by applying different ROI sizes and positions, whole tumour volume measurements were the most reliable [27]. The above-described phenomenon may also explain why the whole-volume ADC measurements resulted in a larger variance and higher standard deviations, which is likely to
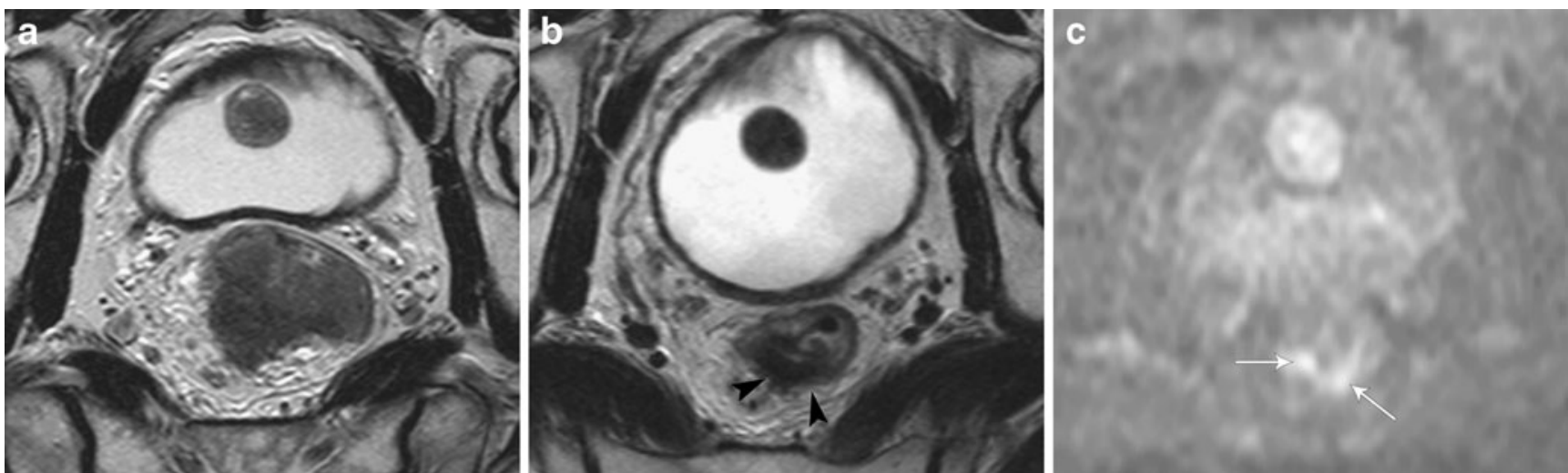

Fig. 5 Axial T2-weighted images of a male patient with a rectal tumour before (a) and after (b) chemoradiation treatment. An ill-defined residual area of hypointense signal intensity, indicative of fibrosis, is visible after CRT (arrowheads). On the corresponding diffusion image (c) there is still an area of high signal intensity, suggestive of residual tumour (arrows). Because of its irregular aspect and ill-defined borders, however, it is difficult to delineate an ROI, explaining the relatively poor interobserver agreement for the post-CRT ADC measurements. At histology, a ypT1 residual tumour was found 
reflect the heterogeneous nature of the tumour, including solid foci, as well as areas of necrosis and fibrosis. Altogether these findings suggest that whole-volume measurements might be a better indicator of tumour viability and may therefore be more suitable for assessment of response. Furthermore, as was also stressed by Goh et al. [27], if variations in ROI substantially influence the measurements, efforts should be made to standardize their application for clinical use. Interestingly, we observed no significant differences in tumour ADC or SD between the whole-volume measurements and the single-slice approach, suggesting that the latter may also be used as a less timeconsuming alternative. However, one should keep in mind that the single-slice method was subject to a much larger interobserver variability and whole-volume measurements thus remain the single most reliable method.

Our study is limited because of its retrospective nature and the relatively small patient numbers. Furthermore, it was sometimes difficult to position regions of interests due to susceptibility artefacts occurring around air-tissue interfaces. This was especially challenging after chemoradiation, in cases where only a limited or no residual tumour could be identified on DWI. Susceptibility artefacts might be minimised by applying rectal wall distension with intraluminal filling, which we have not done in the current study. The specific focus of this study was to determine the effect of ROI size and positioning on tumor ADC evaluation and not to assess the relation between ADC and response, as various previous authors have done [1624]. As such, we chose not to include a correlation between $\mathrm{ADC}$ and histopathological parameters of response.

In conclusion, variations in ROI size and positioning have a significant effect on tumour ADC values and interobserver variability. The most reproducible results are obtained when measuring ADC of the whole tumour volume. Interobserver variability is larger after chemoradiation treatment than before. These issues should be taken into account when considering the use of ADC as a potential biomarker for response in clinical practice.

Open Access This article is distributed under the terms of the Creative Commons Attribution Noncommercial License which permits any noncommercial use, distribution, and reproduction in any medium, provided the original author(s) and source are credited.

\section{References}

1. Habr-Gama A, Perez RO, Proscurshim I, Canpos FG, Nadalin W, Kiss D, Gama-Rodrigues J (2006) Patterns of failure and survival for nonoperative treatment of stage $\mathrm{c} 0$ distal rectal cancer following neoadjuvant chemoradiation therapy. J Gastrointest Surg 10:1319-1328, discussion 1328-1319
2. Lezoche G, Baldarelli M, Guerrieri M, Paganini AM, De Sanctis A, Bartolacci S, Lezoche E (2008) A prospective randomized study with a 5-year minimum follow-up evaluation of transanal endoscopic microsurgery versus laparoscopic total mesorectal excision after neoadjuvant therapy. Surg Endosc 22:352-358

3. Maas M, Nelemans PJ, Valentini V, Das P, Rödel C, Kuo LJ, Calvo FA, García-Aguilar J, Glynne-Jones R, Haustermans K, Mohiuddin M, Pucciarelli S, Small W Jr, Suárez J, Theodoropoulos G, Biondo S, Beets-Tan RG, Beets GL (2010) Long-term outcome in patients with a pathological complete response after chemoradiation for rectal cancer: a pooled analysis of individual patient data. Lancet Oncol $11: 835-844$

4. Barbaro B, Vitale R, Leccisotti L, Vecchio FM, Santoro L, Valentini V, Coco C, Pacelli F, Crucitti A, Persiani R, Bonomo L (2010) Restaging locally advanced rectal cancer with MR imaging after chemoradiation therapy. Radiographics 30:699-716

5. Capirci C, Rubello D, Chierichetti F, Crepaldi G, Carpi A, Nicolini A, Mandoliti G, Polico C (2004) Restaging after neoadjuvant chemoradiotherapy for rectal adenocarcinoma: role of F18-FDG PET. Biomed Pharmacother 58:451-457

6. Bammer R (2003) Basic principles of diffusion-weighted imaging. Eur J Radiol 45:169-184

7. Koh DM, Collins DJ (2007) Diffusion-weighted MRI in the body: applications and challenges in oncology. AJR Am J Roentgenol 188:1622-1635

8. Bruegel M, Holzapfel K, Gaa J, Woertler K, Waldt S, Kiefer B, Stemmer A, Ganter C, Rummeny EJ (2008) Characterization of focal liver lesions by ADC measurements using a respiratory triggered diffusion-weighted single-shot echo-planar MR imaging technique. Eur Radiol 18:477-485

9. Lim HK, Kim JK, Kim KA, Cho KS (2009) Prostate cancer: apparent diffusion coefficient map with T2-weighted images for detection - a multireader study. Radiology 250:145-151

10. Patterson DM, Padhani AR, Collins DJ (2008) Technology insight: water diffusion MRI-a potential new biomarker of response to cancer therapy. Nat Clin Pract Oncol 5:220-233

11. Padhani AR, Liu G, Koh DM, Chenevert TL, Thoeny HC, Takahara T, Dzik-Jurasz A, Ross BD, Van Cauteren M, Collins D, Hammoud DA, Rustin GJ, Taouli B, Choyke PL (2009) Diffusionweighted magnetic resonance imaging as a cancer biomarker: consensus and recommendations. Neoplasia 11:102-125

12. Theilmann RJ, Borders R, Trouard TP, Xia G, Outwater E, Ranger-Moore J, Gillies RJ, Stopeck A (2004) Changes in water mobility measured by diffusion MRI predict response of metastatic breast cancer to chemotherapy. Neoplasia 6:831-837

13. Cui Y, Zhang XP, Sun YS, Tang L, Shen L (2008) Apparent diffusion coefficient: potential imaging biomarker for prediction and early detection of response to chemotherapy in hepatic metastases. Radiology 248:894-900

14. Koh DM, Scurr E, Collins D, Kanber B, Norman A, Leach MU, Husband JE (2007) Predicting response of colorectal hepatic metastasis: value of pretreatment apparent diffusion coefficients. AJR Am J Roentgenol 188:1001-1008

15. Jain R, Scarpace LM, Ellika S, Torcuator R, Schultz LR, Hearshen D, Mikkelsen T (2010) Imaging response criteria for recurrent gliomas treated with bevacizumab: role of diffusion weighted imaging as an imaging biomarker. J Neurooncol 96:423-431

16. Dzik-Jurasz A, Domenig C, George M, Wolber J, Padhani A, Brown G, Doran S (2002) Diffusion MRI for prediction of response of rectal cancer to chemoradiation. Lancet 360:307308

17. Sun YS, Zhang XP, Tang L, Ji JF, Gu J, Cai Y, Zhang XY (2010) Locally advanced rectal carcinoma treated with preoperative chemotherapy and radiation therapy: preliminary analysis of diffusion-weighted MR imaging for early detection of tumor histopathologic downstaging. Radiology 254:170-178 
18. Roth Y, Tichler T, Kostenich G, Ruiz-Cabello J, Maier SE, Cohen JS, Orenstein A, Mardor Y (2004) High-b-value diffusionweighted MR imaging for pretreatment prediction and early monitoring of tumor response to therapy in mice. Radiology 232:685-692

19. Lambrecht M, Deroose C, Roels S, Vandecaveye V, Penninckx F, Sagaert X, van Cutsem E, de Keyzer F, Haustermans K (2010) The use of FDG-PET/CT and diffusion-weighted magnetic resonance imaging for response prediction before, during and after preoperative chemoradiotherapy for rectal cancer. Acta Oncol 49:956-963

20. Kremser C, Judmaier W, Hein P, Griebel J, Lukas P, de Vries A (2003) Preliminary results on the influence of chemoradiation on apparent diffusion coefficients of primary rectal carcinoma measured by magnetic resonance imaging. Strahlenther Onkol 179:641-649

21. Hein PA, Kremser C, Judmaier W, Griebel J, Pfeiffer KP, Kreczy A, Hug EB, Lukas B, DeVries AF (2003) Diffusion-weighted magnetic resonance imaging for monitoring diffusion changes in rectal carcinoma during combined, preoperative chemoradiation: preliminary results of a prospective study. Eur J Radiol 45:214-222

22. Kim SH, Lee JY, Lee JM, Han JK, Choi BI (2011) Apparent diffusion coefficient for evaluating tumour response to neoadjuvant chemoradiation therapy for locally advanced rectal cancer. Eur Radiol 21:987-995
23. Kim SH, Lee JM, Hong SH, Kim GH, Lee JY, Han JK, Choi BI (2009) Locally advanced rectal cancer: added value of diffusionweighted MR imaging in the evaluation of tumor response to neoadjuvant chemo- and radiation therapy. Radiology 253:116125

24. Seierstad T, Roe K, Olsen DR (2007) Noninvasive monitoring of radiation-induced treatment response using proton magnetic resonance spectroscopy and diffusion-weighted magnetic resonance imaging in a colorectal tumor model. Radiother Oncol $85: 187-194$

25. Woodhams R, Kakita S, Hata H, Iwabuchi K, Umeoka S, Mountford CE, Hatabu H (2009) Diffusion-weighted imaging of mucinous carcinoma of the breast: evaluation of apparent diffusion coefficient and signal intensity in correlation with histologic findings. AJR Am J Roentgenol 193:260-266

26. Takahara T, Imai Y, Yamashita T, Yasuda S, Nasu S, Van Cauteren M (2004) Diffusion weighted whole body imaging with background body signal suppression (DWIBS): technical improvement using free breathing, STIR and high resolution 3D display. Radiat Med 22:275-282

27. Goh V, Halligan S, Gharpuray A, Wellsted D, Sundin J, Bartram CI (2008) Quantitative assessment of colorectal cancer tumor vascular parameters by using perfusion $\mathrm{CT}$ : influence of tumor region of interest. Radiology 247:726-732 